\title{
BMJ Open Protocol for a prospective observational study to improve prehospital notification of injured patients presenting to trauma centres in India
}

\author{
Biswadev Mitra, ${ }^{1,2,3}$ Joseph Mathew, ${ }^{1,2}$ Amit Gupta, ${ }^{4}$ Peter Cameron,,${ }^{1,2,3}$ \\ Gerard O'Reilly, ${ }^{1,2,3}$ Kapil Dev Soni, ${ }^{4}$ Gaurav Kaushik, ${ }^{4}$ Teresa Howard, ${ }^{1}$ \\ Madonna Fahey, ${ }^{1}$ Michael Stephenson, ${ }^{5,6}$ Vineet Kumar, ${ }^{7}$ Sharad Vyas, ${ }^{8}$ \\ Satish Dharap, ${ }^{7}$ Pankaj Patel, ${ }^{9}$ Advait Thakor, ${ }^{10}$ Naveen Sharma, ${ }^{4}$ Tony Walker, ${ }^{5}$ \\ Mahesh Chandra Misra, ${ }^{11}$ Russell Gruen, ${ }^{12}$ Mark Fitzgerald, ${ }^{1}$ on behalf of the \\ Australia-India Trauma System Collaboration
}

To cite: Mitra B, Mathew J, Gupta A, et al. Protocol for a prospective observational study to improve prehospital notification of injured patients presenting to trauma centres in India. BMJ Open 2017;7:e014073. doi:10.1136/ bmjopen-2016-014073

- Prepublication history for this paper is available online. To view these files please visit the journal online (http://dx.doi org/10.1136/bmjopen-2016014073).

Received 29 August 2016 Revised 13 April 2017 Accepted 17 May 2017

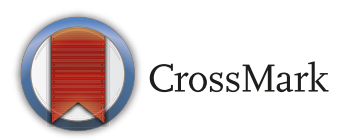

For numbered affiliations see end of article.

Correspondence to Dr Biswadev Mitra; biswadev.mitra@monash.edu

\section{ABSTRACT}

Introduction Prehospital notification of injured patients enables prompt and timely care in hospital through adequate preparation of trauma teams, space, equipment and consumables necessary for resuscitation, and may improve outcomes. In India, anecdotal reports suggest that prehospital notification, in those few places where it occurs, is unstructured and not linked to a well-defined hospital response. The aim of this manuscript is to describe, in detail, a study protocol for the evaluation of a formalised approach to prehospital notification.

Methods and analysis This is a longitudinal prospective cohort study of injured patients being transported by ambulance to major trauma centres in India. In the preintervention phase, prospective data on patients will be collected on prehospital assessment, notification, inhospital assessment, management and outcomes and recorded in a new tailored multihospital trauma registry. All injured patients arriving by ambulance and allocated to a red or yellow priority category will be eligible for inclusion. The intervention will be a prehospital notification application to be used by ambulance clinicians to notify emergency departments of the impending arrival of a patient. The proportion of eligible patients arriving to hospital after notification will be the primary outcome measure. Secondary outcomes evaluated will be availability of a trauma cubicle, presence of a trauma team on patient arrival, time to first chest X-ray and inhospital mortality. Progress Ethical approval has been obtained from the All India Institute of Medical Sciences, New Delhi and sitespecific approval granted by relevant trauma services. The trial has also been registered with the Monash University Human Research and Ethics Committee; Project number: CF16/1814 - 2016000929. Results will be fed back to prehospital and hospital clinicians via a series of reports and presentations. These will be used to facilitate discussions about service redesign and implementation. It is expected that evidence for improved outcomes will enable widespread adoption of this intervention among centres in all settings with less established tools for prehospital assessment and notification.

\section{Strengths and limitations of this study}

- This study attempts to address the burden of trauma in a setting with high incidence of injuries and highmortality rates.

- Most deaths occur prehospital and interventions in this setting have the potential to deliver the greatest benefit.

- This is the first study on implementation and evaluation of a structured prehospital notification system in India.

- This study includes only patients transported by ambulance to four trauma centres in India, and thus will capture only a small proportion of the population at risk.

- Outcomes are limited to inhospital analysis, and further studies will be required to assess longer term functional outcomes of injured patients.

Trial registration number NCT02877342; Pre-results.

\section{BACKGROUND}

There is now growing evidence that providing trauma care within a well-organised system saves many lives and prevents long-term disability. ${ }^{1-3}$ The basis of a well-organised trauma system is an agreed trauma triage process at each step along the patient journey. The correct level of prehospital response is based on specified criteria. This is then followed by transfer to an appropriate facility based on patient, mechanistic and geographical data. The hospital response on patient arrival is based on this prehospital data with additional information about prehospital treatment and response to early treatment.

In major trauma resuscitation, management of immediately life-threatening injuries 
requires rapid identification and management of threats to airway, breathing, circulation and brain function. With sufficient notice, trauma hospitals can usually mobilise a team with relevant expertise, which may involve an anaesthetist, surgeon and emergency physician, specialist nurses and a radiographer, to be present and ready for patient arrival. Prehospital notification alone has been found to be independently associated with reduced mortality in trauma centres. ${ }^{4}$ Some notification is common in highly developed trauma systems, usually by radio to the triage desk of the receiving hospital, although standards have not been set for the format or content of communicated information.

In India, patients are brought to hospital by various means, for example, in Delhi $42 \%$ of the 9021 seriously injured were transported by Delhi Police, whereas only $2 \%$ were transported by ambulance in $2014 .{ }^{5}$ Prehospital notification is rare but is theoretically possible because ambulance and police usually carry very high-frequency radio units. Most prehospital and hospital staff carry personal mobile phones that may also be used for communication. In addition to expediting specialist care on arrival to hospital, prehospital communication also enables information sharing from the trauma centre to the caller (such as 'ensure the airway is clear' or 'if you can see the bleeding site, apply pressure'), which may be valuable for variably trained non-medical transporters.

The primary aim of this project is to evaluate the effectiveness of a protocolised prehospital notification system using existing smartphones to improve the rate of prehospital notification of injured patients arriving to major trauma centres. Secondary aims were to assess the effect of this intervention on the availability of an appropriate trauma cubicle on patient arrival, the formation of an appropriate receiving trauma team, time to first radiological imaging and inhospital mortality.

\section{METHODS}

Setting

The study will be conducted in four major trauma centres in India. In India, any teaching hospital serviced by specialty departments of general surgery, orthopaedics and neurosurgery are considered to be major trauma centres. The Jai Prakash Narayan Apex Trauma Center, All India Institute of Medical Sciences (AIIMS), New Delhi admits approximately 4000 severely injured (ISS $>12$ ) patients per year. Guru Tegh Bahadur (GTB) Hospital is a 1500-bed hospital situated in the National Capital Region of Delhi, India, with a trauma census of approximately 1500 patients per year with limb or life-threatening injuries. The Lokmanya Tilak Municipal General (LTMG) Hospital is a level 1 trauma centre, which caters to the megapolis of Mumbai and receives about 2500 patients with limb or life-threatening injuries per year. The Sheth Vadilal Sarabhai General (VS) Hospital caters to areas in and around Ahmedabad with an average annual admission secondary to limb or life-threatening injuries of about 1200 per year.

\section{Study design}

This is a prospective cohort study of injured patients being transported by ambulance to the major trauma centre study sites. The preintervention phase will collect data on current practice (observation of current practice and data collection) and will be compared with the intervention phase after the introduction of prehospital notification through smartphones.

\section{Study population/recruitment procedure}

All injured patients arriving by ambulance and allocated to a red (first) or yellow (second) priority category will be eligible for inclusion. Data will be collected prospectively by trained data collectors positioned in the trauma centres. Prehospital data will be abstracted by data collectors from ambulance worksheets at the time of patient arrival. All data will be entered into a trauma registry and patients will be followed up to hospital discharge. A flow chart of the study population is illustrated in figure 1 .

Retrospective inclusion in the registry will be continued for all screened patients presenting to any of the included hospitals with injury (including near drowning) as the primary diagnosis and with at least one of the following criteria:

1. Admission to hospital,

2. Death after triage but before admission,

3. Dead on arrival.

\section{Exclusion criteria}

Patients meeting screening criteria will be subsequently excluded from the registry if they meet any of the following criteria:

1. Dead at scene (ie, not transported to hospital),

2. Alive at triage but not admitted to hospital (discharged alive without hospital admission),

3. Isolated poisoning,

4. Isolated burns,

5. Single-digit finger or toe amputations (unless of the thumb or great toe) only.

Data on injured patients arriving by other means, for example, private car or police will be included in the registry and numbers and outcomes reported, but not analysed as an outcome of the intervention, as the intervention would not be available to such patients as part of this study.

\section{Intervention}

The prehospital notification application (named Suchana) is an android application that will be used by ambulance and emergency clinicians in India to notify emergency departments of selected hospitals of an impending arrival of a patient requiring advanced lifesaving assistance. Prehospital clinicians will receive training on use of this application which will be accessible from personal smartphones. The application will use a simple algorithm based on trauma triage principles 


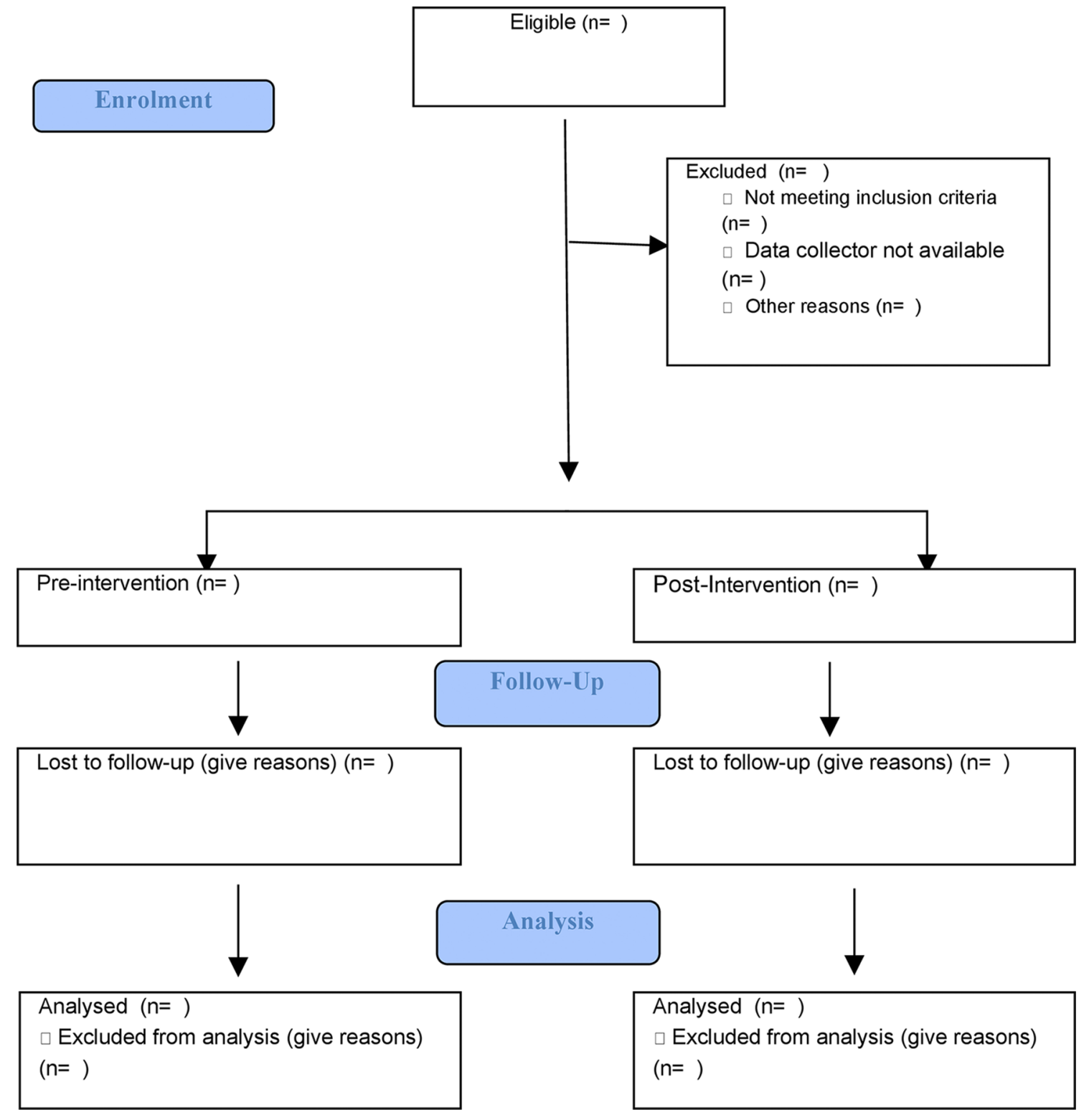

Figure 1 Patient recruitment flow diagram.

developed by the Australia India Trauma Systems Collaboration (AITSC).

The Suchana application will be accessed by emergency medical technician via a unique login and device registration. No identifying information is collected by the application with an incident identification number linking the application data to the AITSC Trauma Registry, which is hosted on a secure hospital server in Delhi. The network traffic between the application and the hospital server is Secure Sockets Layer encrypted with hypertext transfer protocol secure-based protocols.

The prehospital notification application will be able to collect basic patient identifiers and using an algorithm designed by the AITSC, push derived information to designated receivers. The prehospital triage process for injured patients was collaboratively developed using a combination of the current AIIMS trauma flag system (figure 2), the Field Triage Decision Scheme developed by the American College of Surgeons and guidelines for trauma triage at The Alfred Hospital Emergency and Trauma Centre, Victoria, Australia. ${ }^{36}$ Data points were determined after taking into account the variation in training among prehospital clinicians in the study setting, avoiding criteria that require a high level of clinical judgement. The final algorithm is presented in table 1 and on the basis of selected criteria, classifies patients to three priorities for trauma resuscitation.

Priority 1 (red): Serious life-threatening injury/illness.

Victims with life-threatening injuries or illness (such as head injuries, severe burns, severe bleeding, cardiac arrest, breathing impaired, internal injuries) are assigned a priority 1 or 'red' triage tag code (meaning first priority for treatment).

Priority 2 (yellow): Moderate to serious injury/illness (not immediately life threatening). 


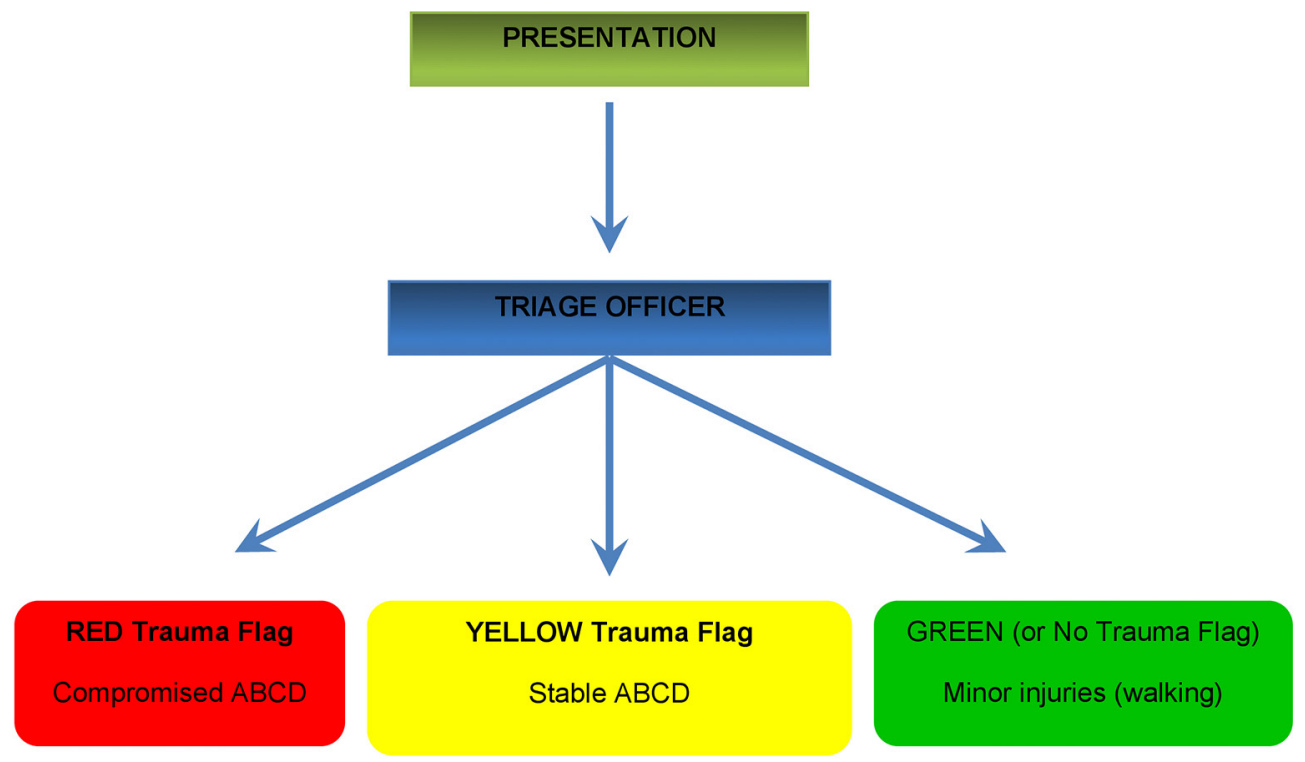

Figure 2 Trauma triage protocol. ABCD: Airway, Breathing, Circulation, Disability.

Victims with potentially serious (but not immediately life-threatening) injuries (such as fractures) are assigned a priority 2 or 'yellow' (meaning second priority for treatment) triage tag code.

Priority 3 (green): 'Walking-wounded' victims who are not seriously injured.

Triaged as 'walking wounded', and a priority 3 or 'green' classification (meaning delayed treatment).

\section{Demographics}

Patient demographics will be collected to define the population and ensure selection of a representative sample. Data collected will include data and time of presentation, age, sex and place of residence.

\section{Exposure variables}

Data collected on prehospital variables will include vital signs-systolic blood pressure (in $\mathrm{mm} \mathrm{Hg}$ ), pulse rate (in beats $/ \mathrm{min}$ ), respiratory rate (in breaths/min) and consciousness on the alert, voice, pain, responsive scale. Mechanism and place of injury details will also be collected (table 1). These will be measured by prehospital staff after first contact with the patient. In the prehospital phase, this will be recorded in paper format as per current practice and extracted by study personnel on arrival to hospital. During the intervention phase, these data will be entered directly into the application. The prehospital trauma priority flag (red, yellow or green) will be collected as communicated by hospital staff on arrival to the emergency department or in the intervention phase, as generated by the intervention or communicated by prehospital staff.

Data on demographic and injury characteristics will be collected and include designation of person receiving notification, mechanism and intent of injury and transport type. International Statistical Classification of Diseases and Related Health Problems 10th Revision
(ICD10) External Cause Codes will be collected for activity, location and intent. For Mechanism, ICD10 codes will be aggregated and condensed to improve feasibility, completeness and accuracy. Data on clinical signs will include inhospital vital signs-systolic blood pressure, heart rate, respiratory rate and Glasgow Coma Scale (GCS) with additional data on GCS components and oxygen saturation. Management data collected will include emergency department disposition times, details on inhospital operative procedures, inhospital radiological investigations, neurosurgical consultation and blood transfusions. Further details on injury severity will be collected using the Abbreviated Injury Severity (AIS) scale codes (2005 Update 2008 (AIS 2008)). ${ }^{7}$

\section{Primary outcome}

Prehospital notification will be the primary outcome, defined as a phone call or message to a treating hospital clinician regarding an injured patient en route to hospital. For the purpose of this research project, a patient will satisfy primary outcome criteria if prehospital notification with any information has occurred. This variable will be collected and analysed as a binary variable.

\section{Secondary outcomes}

A trauma callout will be recorded as a binary variablewhether one has occurred or not. Date and time of this callout will also be recorded.

The presence of a trauma team leader at the time of patient arrival will be collected as a binary variable. This is the person whose role is to coordinate the trauma resuscitation (primary and secondary survey) — this is, generally, a hands-off role undertaken by the most senior clinician in the trauma team. The team leader should be someone not already/always present in the area where trauma bay is located and have the ability to lead or make decisions regarding treatment (eg, emergency transfer to operating 
Table 1 Prehospital data collection using smartphone application and generation of trauma priority flag. ${ }^{\S}$

\begin{tabular}{|c|c|}
\hline Demographics & $\begin{array}{l}\text { Age-years } \\
\text { Sex }\end{array}$ \\
\hline Mechanism of injury & $\begin{array}{l}\text { RTI-high speed } \\
\text { RTI pedestrian/bicycle } \\
\text { RTI motorbike } \\
\text { RTI unspecified } \\
\text { Fall from height (10 feet/3 metres) }{ }^{*} \\
\text { Penetrating trauma-stab/gunshot* } \\
\text { Railway incident* } \\
\text { Blunt assault } \\
\text { Near drowning* } \\
\text { Other }\end{array}$ \\
\hline $\begin{array}{l}\text { Serious injury } \\
\text { identification }\end{array}$ & $\begin{array}{l}\text { Penetrating to head, neck, torso* } \\
\text { Chest injury including pneumothorax } \\
\text { Crush injury including degloving }^{\dagger} \\
\text { Amputation proximal to wrist and } \\
\text { ankle* }^{\star} \\
\text { Suspected pelvic injury }^{\dagger} \\
\text { Open or closed suspected skull } \\
\text { fracture* } \\
\text { Spinal injury } \\
\text { Other }\end{array}$ \\
\hline Vital signs & 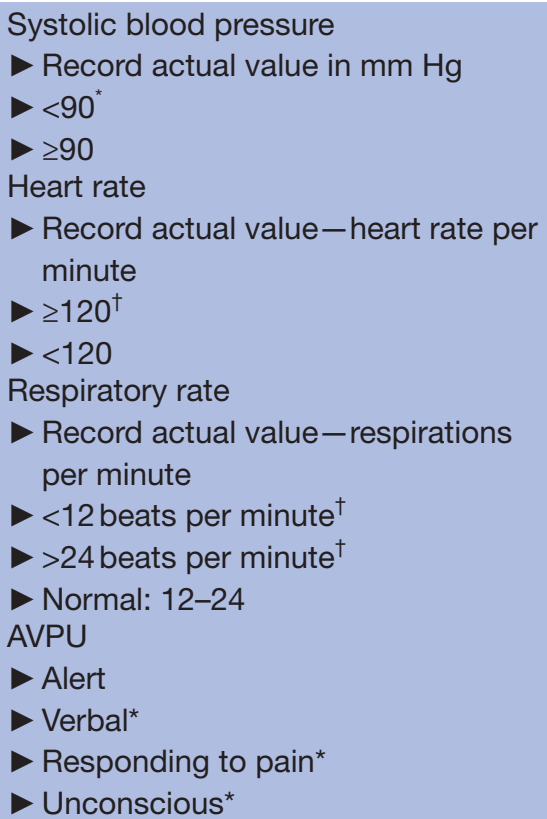 \\
\hline
\end{tabular}

Automatic flag-generation rules.

*Presence of a variable generates a RED flag.

†Presence of two or more variables generates a RED

flag; presence of one variable only generates a YELLOW flag.

§Presence of none of the numbered variables generates a GREEN flag.

AVPU, alert, verbal, pain, unconscious; RTI, road traffic incident.

theatre or not). The leader is expected to be present by the time of patient arrival at the definitive care hospital. The designation of the usual team leader is site specific:

JPN: Senior Resident.

GTBH: Senior Resident.

VSH: Third year Resident.

LTMGH: Third year surgical resident.
Readiness of a trauma bay is defined as at least one trauma bay that has been allocated and empty (therefore ready to receive a patient) on arrival. Time at which the first chest X-ray commenced will be recorded. The location to which a patient was discharged on completion of hospital management will be recorded and the secondary outcome for inhospital death collected as a binary variable.

Predefined subgroup analyses will be conducted on each centre, severity of injury on presentation, that is, 'red' and 'yellow' category and patients who are declared dead on arrival.

\section{Data analysis}

Continuous data will be summarised using mean with SD if normally or near-normally distributed or with medians and interquartile ranges for skewed data. Ordinal data will be summarised using medians and interquartile ranges. Nominal, including binary, data will be presented as counts with proportions within their categories. Statistical significance will be defined as $\mathrm{p}<0.05$. A difference between means will be analysed for statistical significance using the Student's t-test while the Wilcoxon rank sum test will be used for assessing statistical significance for a difference between medians. The $\chi^{2}$ test or Fisher's exact test will be used for nominal data according to cell frequencies. All analyses will be performed using StataV.12.0.

\section{Sample size}

The proportion of patients achieving the primary outcome after intervention was targeted to be 0.70 (achieved by consensus among trauma leaders in Australia and India). This was considered to be a clinically significant level compared with anecdotal reports of current practice at extremely low rates of prehospital notification.

The minimum acceptable difference from this hypothesised ideal proportion was considered to be $10 \%$, with a one-sided absolute difference of $>10 \%$ (ie, less than $0.6)$ to be considered as failure to achieve the primary outcome. The estimated sample size for a one-sample comparison of proportion with the hypothesised value of 0.70 , an alpha of 0.05 and power of 0.90 was 191 after intervention.

\section{Funding}

This project is part of the AITSC that brings together public and private sector clinicians and researchers to improve information, resources and pilot new systems of care. Commencing in 2013, the Australian and Indian governments invested through their Australia-India Strategic Research Fund Grand Challenge Scheme, to find the best ways of delivering needed care to injured people. The AITSC is funded through the former Grand Challenge Fund. Australia's Department of Industry, Innovation and Science and the Government of India's Department of Science and Technology jointly manage the Grand Challenge Fund. It is expected that these 
projects will lay the foundations for a national trauma system in India and improved trauma care in much of Australia. They will also provide needed evidence about low-cost trauma system interventions that could be implemented in most countries without the need for major health system redesign.

\section{Ethics and dissemination}

Although this study primarily involves service evaluation, ethical approval was obtained to allow collection of patient identifiers. Therefore, full ethical approval has been obtained from the All India Institute of Medical Sciences (AIIMS), New Delhi, India, and site-specific approval has been acquired from the relevant trauma services. The project has been reviewed by the Monash University Human Research Ethics Committee and approval granted; Project number: CF16/1814 - 2016000929. Study results will be disseminated among prehospital and hospital clinicians across participating sites. It is expected that ongoing evaluation will inform sustainability of the proposal and requirements for refreshers. It is expected that study findings will be presented at scientific congresses and published in peer-reviewed manuscripts. The protocol is registered at clinicaltrial.gov, ID No: NCT02877342.

\section{DISCUSSION}

Currently, injury in India is a leading cause of years of productive life lost and the leading cause of death for those under 35 years old. This national injury burden is growing and the ongoing rise in the trauma burden is mostly in the form of road traffic crashes. India has $1 \%$ of the total vehicles in the world yet accounts for $6 \%$ of total road accidents globally. It is estimated that there are 400000 road traffic crashes in India each year, resulting in 100000 deaths and 1.2 million individuals who are seriously injured. The burden is borne disproportionately by young people with a regional report concluding a total of 6134 life years were lost each year in a population of 108 000 following unintentional injuries. ${ }^{8}$

International establishment of trauma systems incorporating centralised trauma centres has helped address the injury burden by providing prompt, specialist trauma care. In such systems, effective therapy for the severely injured is facilitated by an interdisciplinary and integrated (horizontal) approach to undifferentiated trauma with input from prehospital and inhospital resuscitation teams. ${ }^{9}$

This will be the first study to assess utilisation of a smartphone-based intervention to improve prehospital notification. Smartphones currently include all the features of a laptop, including web browsing, Wi-Fi and third-party application. Currently, the most popular smartphones are Google's Android, Apple's iOS mobile operating systems and Nokia-X series. As well as being technologically advanced, India's adoption of smartphones and tablets has been high and continues to rapidly grow. Doctors in emergency departments have smartphones and ambulance services have access to smartphones and/or tablets in their ambulances. Similar technology has been successfully used to monitor patients with chronic health needs and self-examination. ${ }^{10}$ There is currently no standardised system of prehospital notification in India for injured patients. The public ambulance system in India is rapidly expanding, adopting computerised systems of global positioning system tracking and call allocation similar to high-income countries.

Prehospital services are rapidly expanding across India with over 20 states with some form of government or private-public partnership prehospital/emergency service. India is rapidly moving towards a Western model of prehospital service with the aim that all major trauma patients will arrive by ambulance staffed by personnel trained in basic or advanced life support. Our project is directed towards the ambulance services, while other groups such as the SaveLIFE Foundation, an independent, non-profit, non-governmental organisation focused on improving road safety and emergency medical care across India, who have introduced a training programme for police as first responders. Other groups that have introduced similar programmes in India are the Society of Indian Automotive Manufacturers and the International Road Federation in Geneva.

Additional data from this project may identify potential benefits from new approaches to communication between prehospital care providers (ambulance, emergency and police services) and receiving hospitals before the patient arrives. As this project will use standardised, locally tailored protocols for notifying and responding to a seriously injured person who is being brought to hospital, there is further scope for validating such protocols for more widespread use. The current scope of the project and funding precludes training and evaluation of non-ambulance care providers and expected success of this project will likely provide the impetus to introduce prehospital notification more widely across the country.

Previous studies evaluating trauma outcomes in India have been limited with absence of prehospital data and inability to evaluate the influence of prehospital factors on trauma outcomes. ${ }^{11}$ This intervention and the accompanying registry will be among the first to include robust prehospital data, collected prospectively, in evaluating trauma outcomes. Trauma registries are integral to trauma system improvement. In low/middle-income countries, where the burden of injury is much greater, the known activity of trauma registries is much less. ${ }^{12}$

This project is fully funded and has progressed to development of the smartphone application and training of data collectors. Relevant ethics committee approvals as described above have been obtained. Preintervention data collection commenced in May 2016. The intervention will commence in February 2017, with expected completion of the project by May 2017. 


\section{CONCLUSIONS}

Routine prehospital notification of injured patients to the receiving trauma centre is currently not practised in India. This study will prospectively record the current practice of prehospital notification, management across prehospital and hospital phases and outcomes of injured patients presenting to four major trauma centres in India. We aim to develop a smartphone application, incorporating essential information for accurate triage of injured patients. The effect of this intervention on prehospital notification rates, along with overall trauma processes and outcomes will be evaluated in a prospective observational study. It is expected that successful evaluation will enable widespread adoption of this intervention in India and other countries.

\section{Author affiliations}

${ }^{1}$ National Trauma Research Institute, Monash University, Melbourne, Victoria, Australia

${ }^{2}$ Emergency \& Trauma Centre, The Alfred Hospital, Melbourne, Victoria, Australia ${ }^{3}$ Department of Epidemiology and Preventive Medicine, Monash University, Melbourne, Victoria, Australia

${ }^{4}$ Division of Trauma Surgery and Critical Care, All India Institute of Medical Sciences, New Delhi, Delhi, India

${ }^{5}$ Executive Team, Ambulance Victoria, Doncaster, Victoria, Australia

${ }^{6}$ Department of Community Emergency Health and Paramedic practice, Monash University, Melbourne, Victoria, Australia

${ }^{7}$ Surgery, Lokmanya Tilak Municipal Medical College and General Hospital, Mumbai, Maharashtra, India

${ }^{8}$ Department of Surgery, BJ Medical College, Ahmedabad, Gujarat, India

${ }^{9}$ Orthopaedic Surgery, Smt NHL Municipal Medical College, Ahmedabad, Gujarat, India

${ }^{10}$ Department of Emergency Medicine, Smt NHL Municipal Medical College, Ahmedabad, Gujarat, India

${ }^{11}$ The President Cum VICE CHANCELLOR DESIGNATE And Director Of Surgical Disciplines, Mahatma Gandhi University Of Medical Sciences and Technology, Jaipur, Rajasthan, India

${ }^{12}$ Department of Surgery, Nanyang Technological University, Singapore, Singapore

Contributors The project was planned by RG, MF, MCM, AG and AT. Study design was undertaken, in consultation with all authors, by BM, PC, TH, MF, VK and JM. Site-specific processes were arranged and processes unique to each site added to the manuscript by KDS, GK, SD and PP. Biostatistician G0'R reviewed the proposal and provided input to research design and analysis. MS and TW provided expert opinion on prehospital systems. All authors reviewed and edited the final manuscript.
Competing interests None declared.

Ethics approval All India Institute of Medical Sciences, New Delhi, India; Monash University Research and Ethics Committee.

Provenance and peer review Not commissioned; externally peer reviewed.

Open Access This is an Open Access article distributed in accordance with the Creative Commons Attribution Non Commercial (CC BY-NC 4.0) license, which permits others to distribute, remix, adapt, build upon this work non-commercially, and license their derivative works on different terms, provided the original work is properly cited and the use is non-commercial. See: http://creativecommons.org/ licenses/by-nc/4.0/

C) Article author(s) (or their employer(s) unless otherwise stated in the text of the article) 2017. All rights reserved. No commercial use is permitted unless otherwise expressly granted.

\section{REFERENCES}

1. Cameron PA, Gabbe BJ, Cooper DJ, et al. A statewide system of trauma care in Victoria: effect on patient survival. Med J Aust 2008;189:546-50.

2. MacKenzie EJ, Rivara FP, Jurkovich GJ, et al. A national evaluation of the effect of trauma-center care on mortality. $N$ Engl J Med 2006;354:366-78.

3. Gabbe BJ, Simpson PM, Sutherland AM, et al. Improved functional outcomes for major trauma patients in a regionalized, inclusive trauma system. Ann Surg 2012;255:1009-15.

4. Cameron PA, Gabbe BJ, Smith $\mathrm{K}$, et al. Triaging the right patient to the right place in the shortest time. Br J Anaesth 2014;113:226-33.

5. Gupta A, Misra MC, Kumar S, et al. Centre JAT, editor. Hospital Injury Surveillance and Trauma Registry Report 2011-2012. New Delhi: New Delhi Publications, 2013.

6. Sasser SM, Hunt RC, Faul M, et al. Guidelines for field triage of injured patients: recommendations of the National Expert Panel on Field Triage, 2011. MMWR Recomm Rep 2012;61:1-20.

7. Tohira H, Jacobs I, Mountain D, et al. Comparisons of the outcome prediction performance of injury severity scoring tools using the abbreviated injury scale 90 update 98 (AIS 98) and 2005 update 2008 (AIS 2008). Ann Adv Automot Med 2011;55:255-65.

8. Bose A, Konradsen F, John J, et al. Mortality rate and years of life lost from unintentional injury and suicide in South India. Trop Med Int Health 2006;11:1553-6.

9. Fitzgerald M, Jamieson J, Tee JW, et al. Trauma systems development challenges the conventional medical hierarchy. The Indian Journal of Neurotrauma 2011;8:67-70.

10. Triantafyllidis A, Velardo $\mathrm{C}$, Chantler $\mathrm{T}$, et al. A personalised mobilebased home monitoring system for heart failure: the SUPPORT-HF study. Int J Med Inform 2015;84:743-53.

11. Murlidhar V, Roy N. Measuring trauma outcomes in India: an analysis based on TRISS methodology in a Mumbai university hospital. Injury 2004;35:386-90.

12. O'Reilly GM, Joshipura M, Cameron PA, et al. Trauma registries in developing countries: a review of the published experience. Injury 2013;44:713-21. 